\title{
Why Value is Everything: A User-Centered Approach to Internet Quality of Service and Pricing
}

\author{
Anna Bouch and M. Angela Sasse \\ Department of Computer Science, University College London, Gower Street, London \\ WC1E 6BT, UK \\ $\{$ A.Bouch, A. Sasse@cs.ucl.ac.uk\}
}

\begin{abstract}
To create acceptable levels of Quality of Service (QoS), designers need to be able to predict users' behaviour in response to different levels of QoS. However, predicting behaviour requires an understanding of users' requirements for specific tasks and contexts. This paper reports qualitative and experimental research that demonstrates that future network service must be based on an old principle: service and its associate cost must represent value in terms of the contribution it makes to customers' goals. Human Computer Interaction (HCI) methods can be applied to identify users' goals and associated QoS requirements. Firstly, we used a qualitative approach to establish the mental concepts that users apply when assessing network services and charges. The subsequent experimental study shows that users' require certain types of feedback at the user interface to predict future levels of quality. Price alone cannot be used to regulate demand for QoS.
\end{abstract}

\section{Introduction}

The number of Internet users is expected to triple between 1998 and 2002 [1], largely because of new applications (such as videoconferencing) and new services (such as ecommerce). This shift in usage imposes higher Quality of Service (QoS) requirements at different levels of granularity. It also means that the traditional Internet way of managing quality (best-effort) has to be replaced by a more service-oriented approach, and that service providers need to find a way of creating revenue.

Traffic produced from different applications can be characterized through an associated payment [2],[3],[4]. For example, high-volume video may be prioritized by associating it with a high-price. The majority of pricing schemes are based on the assumption that the amount and type of quality that is detectable within the network is identical to the quality that will be paid for by users. However, the design of sociotechnical environments, such as the evolving Internet, cannot be solely be based on technical considerations. By definition, integration of the requirements of users and technology has to take place.

There are several stakeholders in the design of Internet services: server designers, network providers, advertisers, companies whose products are sold on-line, and consumers themselves. While a vision of the future Internet offers the potential to break traditional barriers in communications and commerce, the current level of 
service does not satisfy the requirements of many users [5],[1]. Failure to understand users' QoS requirements may affect users' conception of a company's stature and commercial viability which, in turn, affects the business interests of service providers and advertisers [6]. The future Internet will have more users and support a greater diversity of Internet applications. It has the potential to change the way that consumers interact with companies. Research is needed to identify users' requirements for QoS and the schemes that are used to charge them for it. Only through such identification will it be possible to achieve the customer satisfaction that leads to the success of any commercial system.

The aim of this research is to define users' requirements for network QoS and charging, and investigate the relationship between users' assessment of QoS in different contexts of use, and the mental models that motivate that assessment. The ultimate aim of the research is to provide models that can be used to predict users' demand for QoS in priced situations. The work reported establishes users' requirements, and what network service designers need to know to make design decisions that support users' goals. The developed models should aid the integration of users' requirements for QoS into systems design, and therefore have immediate practical benefits for systems designers and users themselves. The study reported in this paper was structured in 2 stages:

1) Construct conceptual models that motivate users' perceptions of network QoS and charging mechanisms.

2) Capture users' evaluations of objective QoS in different contexts.

\section{Previous Results}

\subsection{Predictability}

The effect of applying flat and usage-based charging mechanisms to the telephone service was extensively studied in the late seventies [7]. It was shown that users reduced their usage of the network when faced with usage-based charging. Additionally, customers would choose the flat-rate option regardless of the amount of calls made. These results indicate that the predictability and simplicity of charges are criteria valued by customers, and because of this they will actually pay more than their usage levels require. In a study of American households, it was shown that families did not want to install phone lines because they were uncertain about the magnitude of bills incurred by usage [8]. These results suggest that an adequate feedback mechanism, informing customers of likely charges, might increase the acceptability of usage-based charges.

Quota charging has been suggested [9]: Quotas for certain important QoS drivers can be bought prior to network use. Users then have a (dynamic) choice whether to use their quota for any particular transaction. From a user's perspective, this scheme combines the predictability of bounded usage with the flexibility to request increased QoS when it is needed. Under the quota scheme, the average rate of service requests are optimal for every user considering the price and anticipated delay.

Predictability is one of the most fundamental QoS drivers from a user's perspective [10]. The concept Risk Assessment is directly linked with Predictability - 
a low-risk situation is one that is predictable. This suggests that users are prepared to accept charging mechanisms that are predictable. However, somewhat contrary to the findings in [7], more recent studies suggest that users prefer to be able to dynamically change the levels of QoS they receive in line with the value given to the task being performed [11]. Therefore, dynamic pricing needs to provide feedback on network congestion, which would enable users to predict the risk involved in making certain payments.

\subsection{Risk}

Users make a Risk Assessment about whether the QoS they will receive represents value for money. To assess Risk, users consider several sub-concepts; the relevance of the different sub-concepts depend on users' level of knowledge and experience. Users' tolerance of certain levels of QoS, and consequently how much QoS they are prepared to pay for, depends on their expectations [11]. This suggests that the network should provide feedback to users, allowing them to make accurate predictions of future quality.

\subsection{Context of Interaction}

Previous research has shown that users judge the acceptability of pricing schemes according to a variety of dimensions [3]. The salience of these dimensions is determined, not by the fact that they are technically implementable, but by their semantic value. In networked multimedia applications in particular, variations in quality at the network level are not directly linked to the subjective assessment of quality received by users [12]. This suggests that users will pay for the QoS they receive in terms of the media quality they require to complete their goal. For example, with real-time video tasks, participants in previous studies mentioned that the ability to manage the video image in terms of operations such as resizing was important [10]. Clearly, users' need to resize the video image depends on the value placed on what is seen in that image.

\section{Qualitative Study}

\subsection{Method}

The phenomena under investigation are complex. The approach of our study was therefore to combine qualitative and experimental research. Qualitative data is needed to investigate users' motivations and conceptual models. We used focus groups to collect data about the acceptability of different charging mechanisms in more detail with users who have the responsibility for payment of line and usage charges. We used grounded theory methods [13], to analyze focus group data. Grounded theory allows characterization of concepts extracted from conversations. The definitions of these concepts can be systematically tested for validity under experimental conditions. 
Grounded theory has been successfully applied in recent HCI research to elicit user perceptions on issues such as security and privacy [14], [15]. To have practical benefits, it must also be shown that these models have direct impact on users behavior when interacting with a system. Experimental data provides this evidence.

Users. The same 30 participants took part in both the focus groups and experimental parts of the study. Participants were selected to have experience in using multimedia applications. The following criteria was also used to select participants:

1. Responsibility for the payment of subscription and/or usage charges for their network.

2. Use of that network for more than 2 hours per week.

3. Use of a mobile phone.

Focus Groups. The focus groups were designed to investigate:

1. How network QoS charging compares to other forms of charging (e.g. mobile phone usage).

2. Whether the method of charging preferred depends on the importance of the user's task.

3. The extent of users' satisfaction with method of charging for mobile phone usage.

4. Attitudes to budgeting, e.g. do participants think of their budget when making calls $v s$. the absolute per-minute charge?

\subsection{Results}

The main results of the focus groups show that absolute price alone is not a good predictor of users' subjective perception of quality. This means that a service provider cannot assume that charging twice as much for providing twice as much speed on a Web-page download doubles the subjective value of quality to users. This is because users' conceptions of quality are influenced by a number of contextual and social factors. Users made the distinction between the need for an awareness of the charge, and control of that charge, the latter being a chance for users to dynamically and directly reflect QoS needs within an interaction.

Peace of Mind. Peace of Mind was found to be the top-level goal in situations where users were aware of the charge being made for the interaction. It describes the state of mind arising from users' ability to commit to a call and not experience unacceptable changes in price or QoS, that would force a re-evaluation of that commitment.

Usage Trade-offs. Where users are not able to control the QoS they receive in a dynamic fashion, they make usage trade-offs in terms of time. Users also make tradeoffs concerning the charging schemes that they use at particular times of day, and for particular tasks. For example:

'I'll think, how important is it for me to make this call now and if it's not important then I won't do it'. 
'If I'm making a long-distance call I won't use a...land-line, I'll use a different scheme, that's cheaper'.

Commitment. Users make commitments to an interaction. They make an up-front assessment of the importance of an interaction, and make trade-offs in terms of whether that call is important enough to make at that time. This means that users do not want to constantly re-evaluate the value of the interaction. The key, therefore, is to provide initial feedback that gains users' confidence in their ability to control QoS. This enhances users' Commitment to the interaction. To illustrate:

'Understanding what's going on, what I'll get and if it will meet what I want...I suppose it's a peace of mind thing. If you tell me what it's going to be like, then I can go ahead and know I'll complete the call without hassle'.

Participants stressed the benefits of using pre-paid packages. A popular example was where the provider refunds the difference between expenditure on the chosen tariff and that on a cheaper tariff, for the users particular type of usage that month. This provides users with the confidence to commit to an interaction under a guarantee that that interaction represents value for money.

Trust. The notion of Trust plays an important role in influencing requirements for dynamic control of QoS and the schemes used to charge for that QoS. Trust is concerned both with users trusting themselves, and the service provider. Allowing users to dynamically control charges for QoS, or making users aware of their expenditure so that they must reassess their Commitment to the interaction encourages users to believe that there is a risk of making an inappropriate assessment of that quality. Users not trusting their ability to re-evaluate appropriately induces unacceptable cognitive load and a sense of anxiety:

'Knowing that all the time how much it's costing, makes be feel paranoid that I'll go over without knowing, I can't judge that right'.

Providing continuous charging feedback, or even making users aware that continuous charging feedback is possible also promotes a sense of mistrust in the service provider. Users feel that their usage is being monitored and this is a violation of their sense of privacy and control over the outcome of their expenditure. 
Critical Periods. Users attach the concept of a Critical Period to the charging scheme. Users set the Critical Period by specifying a Critical Threshold (see below). This allows them to ascribe different values to interactions whilst retaining the Peace of Mind that they will not exceed an upper bound. The type of tariff determines the Critical Period. For example, the Critical Period for a pre-paid tariff on a mobile telephone is the call that might cause the allowance to run out. Users cannot commit to this call because of their inability to predict future levels of service.

Critical Thresholds. The Critical Threshold is an upper price bound specified by the user, although default settings could be applied to interactions associated with the same task. With data networks, users want to be able to predict if their particular transaction is likely to exceed the Critical Threshold that they have selected. They are then able to assess if a Commitment to the interaction should be made. For example:

'Yes, if I could say, "don't let me spend more that five pounds, and tell me if my (Web-page) selection will take me over it”, yep, that would be excellent, I'd know then whether to go ahead'.

Like Critical Periods, the definition of a Critical Threshold is dependent on the type of tariff users have. For example, the Critical Threshold can be specified in terms of time:

'I hate the time when your allowances are about to run out and you might be cut off in an important call...I want it to say "ok, it's that amount of time left", then I know I can get through the whole thing'.

\section{Experimental Study}

The results from the focus groups showed that, from the user's point of view, there is no linear correlation between QoS requested and the price of that QoS. We devised an experiment to test the ability of the model constructed in focus groups to predict users' behavior whilst interacting with a priced, variable quality network. The experiment asks: what type and frequency of network feedback to users require when interacting with a priced network?

\subsection{Method}

Task. The experiment involved listening to a recording of a 10-minute interview in which an actor played the role of a candidate who was interviewed for a university place. During the experiment users were asked to manipulate audio quality (packet loss), using the QUASS slider [16]. To situate the task in a realistic context, video was streamed at maximum quality.

Participants were either told that tasks involve a measure of task completion (measured tasks), or that no measure is required (unmeasured tasks). This distinction is made to manipulate the importance of the task. In the measured scenario, 
participants were asked to answer specific questions concerning the candidate's responses in the interview. All participants took part in both the unmeasured and measured task, and the same material was used for both conditions. The order of administration of conditions is reversed for half of the participants in order to control for the effects of participants' varying expectations. To further encourage participants to select realistic levels of QoS, they were told that they would be able to keep a monetary equivalent of the budget they had left at the end of the experiment.

Tools. The experiment uses software that allows users to choose the levels audio quality they receive. The software allocates a budget to users prior to interaction. Participants saw the following interfaces:

1. INFORMATION: This is a panel of three menus. The menus are labeled Current Values, Set Preferences and Future Predictions. The options selectable from the menus are:

Under Current Values:

- $\quad$ Price: Shows how much is being paid for the quality requested via QUASS.

- Quality: Shows the current quality being received, as a percentage.

- Network State: Shows the current network state. This could be Empty, Congested or Very Congested. This information is color-coded.

- Current Budget: Shows how much money left to spend by showing the Your Budget display, described below.

\section{Under Set Preferences:}

- Keep current quality setting: Keeps the audio quality at the current level. If this is selected the QUASS tool disappears. This setting has to be cancelled to reconfigure the QUASS tool.

- Cancel quality setting: Brings back the QUASS tool to allow control of audio quality.

- Keep current price the maximum: Selecting this means that the participant won't pay more than is currently being paid.

- Cancel maximum price: Allows the price to fluctuate again.

Under Future Predictions:

- Quality: Shows a prediction of the quality likely in the near future.

- Network State: Shows what the network state is likely to be in the near future. This could be Empty, Congested or Very Congested. This information is color-coded.

- Battery Life: Shows a prediction of the number of minutes the participant can carry on before their money runs out, if the current level of quality continues to be requested. It does this by showing the Your Budget display, described below.

1. Your Budget: What this shows depends on whether it is selected from the Future Predictions (Fig. 1) or the Current Values (Fig. 2) menu. On selecting from the Future Predictions menu the display will show if the budget will run before the end of the experiment by showing Not enough time. 
2. Information Display: Shows the information selected by choosing from the menus in INFORMATION.

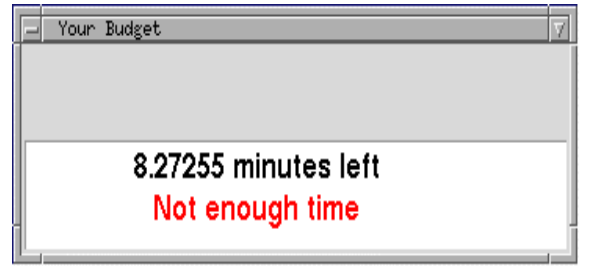

Fig. 1. Your Budget showing Battery Life

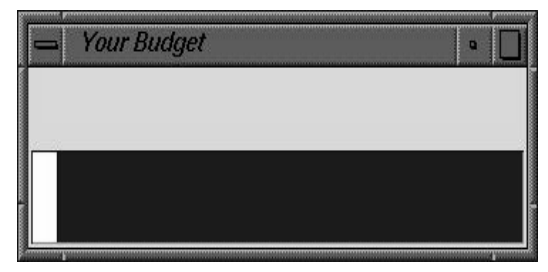

Fig. 2. Your Budget interface showing Current Budget

\section{Experimental Hypotheses.}

- H1: Users will be more likely to select a profile where QoS has priority over price when they are involved in an important task (the 'measured' task).

- H2: Users will be more likely to select a profile where price has priority over QoS when they are involved in a relatively unimportant task (the 'unmeasured' task).

The system used enables users to allow agents to control the levels of QoS received, via profiles. This allows users to specify whether a profile should prioritize delivering a specific level of QoS, or keep within a certain price, in situations where there is a perceived conflict between QoS and price dimensions. It is hypothesized that:

- H3: Users will tolerate lower levels of quality if they select a profile that controls for them.

According to focus group results, the degree to which users relinquish control over their QoS is affected by whether usage at the time is in a Critical Period:

- H4: Users will prioritize price over quality when their budget is relatively low.

Additionally, when QoS is priced, supplying feedback that allows users' to predict and therefore plan their spending affects their requests for QoS.

- H5: Feedback concerning future statistics (e.g. future network congestion) will be requested more frequently than feedback concerning current statistics.

- H6: Feedback concerning 'battery-life' (i.e. displaying how long people have left to interact before their budget runs out) will be the most frequently requested information. 


\subsection{Results}

A strict linear correlation between the amount of quality delivered to users and the price of that quality cannot be assumed. Results show that a number of intervening factors influence whether users consider a certain price appropriate for a particular amount of audio quality. Table 1 presents a summary of results for each experimental hypothesis. These results are discussed below.

Table 1. Results for each experimental hypothesis

\begin{tabular}{|l|c|}
\hline Hypothesis & Result \\
\hline H1 & High task importance = more Quality profile requests \\
\hline H2 & Low task importance = more Price profile requests \\
\hline H3 & Profile selected = lower quality tolerated \\
\hline H4 & Low budget = Price profile prioritized over Quality profile \\
\hline H5 & Future statistics selected more frequently than current statistics \\
\hline H6 & Battery life selected most frequently \\
\hline
\end{tabular}

Users' Tasks. Hypothesis $H 1$ was confirmed by the results of the study. Participants were more likely to select a profile where QoS has priority over price when they were involved in an important task. The frequency with which users selected a QoS profile over a price profile was compared between a measured and unmeasured task scenario.

- Users are more likely to select a profile where QoS has priority over price when they are involved in a relatively important task. This result is significant $(\mathrm{p}<0.05)$.

Using the same methods it was found that hypothesis $H 2$ was not confirmed by the results of the study. This means that:

- Users are not more likely to select a profile where price has priority over QoS when they are involved in a relatively unimportant task.

Fig. 3 shows the feedback required by participants for both measured and unmeasured tasks.

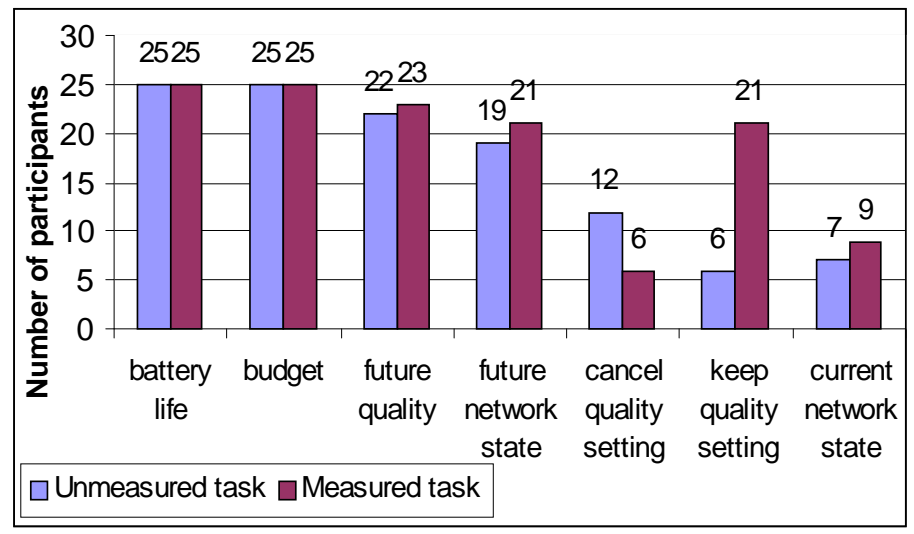

Fig. 3. Number of participants selecting each feedback option 
The levels of Control over QoS. Hypotheses $H 3$ and $H 4$ concerned the levels of quality users would accept depending on the amount of Control they were given over that quality. For each participant who selected a QoS profile, the lowest level of packet loss was compared to the lowest level of packet loss accepted under manual control. Statistical tests reveal that $H 3$ and $H 4$ can be confirmed by the results of the study. This means that:

- Users will tolerate lower levels of quality if they select a profile that controls for them. This result is statistically significant $(\mathrm{p}<0.05)$.

- Users are more likely to prioritize price over quality when their budget is relatively low $(\mathrm{p}<0.05)$.

Predictability. Hypotheses $H 5$ and $H 6$ were confirmed by the results of the study. This means that:

- Users request feedback concerning future statistics more frequently than feedback concerning current statistics.

- Users find information concerning Battery Life the most important type of feedback.

These results can be seen in Fig. 3. Fig. 4 shows the average number of times participants selected certain feedback options. Options that were selected only once have not been included in the analysis, because participants are likely to select options once through curiosity and not through a genuine requirement for feedback. Fig. 4 clearly shows the prevalence of predictive feedback. Statistical analysis shows that Battery Life is selected a significantly higher number of times than other options $(\mathrm{p}<0.05)$.

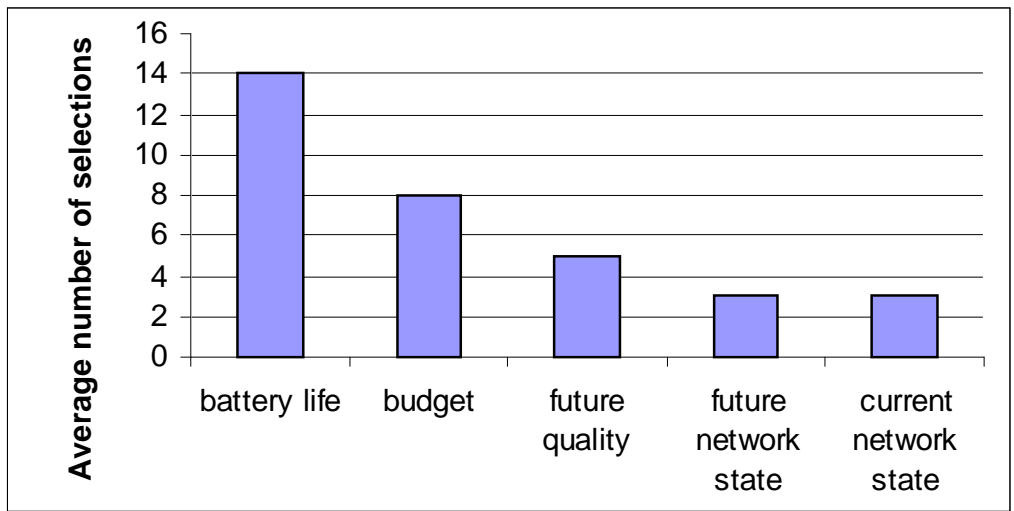

Fig. 4. Average number of selections per option 


\section{Discussion}

Experimental results show that the concepts derived from grounded theory models have an impact on users' behaviour. Profile selection suggests that participants have an understanding that quality is linked to price. Selecting a profile allows users the Peace of Mind that they can concentrate on the task in hand without making reevaluations of the value of received quality. The results show that users make conceptual links between price and quality depending on the context in which they are working. The effect of users' tasks on profile selection is further confirmed by the fact that, in the measured task, participants in this study were more likely to keep the profile selected than to cancel it, compared to those few participants who selected a profile in the unmeasured task. It is likely that participants chose QoS profiles in the measured task scenario to reduce the cognitive load of having to control the quality manually whilst attempting to hear crucial parts of the conversation:

'When I saw it's going to be worse I did try to not ask for as much, to try to get some leeway. But it was more important to hear it, to get the answers'.

Apart from when their budget was relatively full, very few participants selected a profile where price has precedence over quality $(\mathrm{H} 2)$. These results show that the goal of users' interaction has priority over the price levied against that interaction. The confirmation of Hypothesis 4 (H4) shows that there is a complex relationship between users' tasks and the price they pay for the quality that helps them complete those tasks. The potential for price to correlate with QoS demands somewhat depends on whether users choose to use agents to control their QoS rather than to control it manually. This is because fluctuation in demands for QoS is greatly reduced if a profile is used. However, the results reported in this paper have shown that the importance of users' tasks varies according to their amount and rate of expenditure. This is shown by prioritizations of price over quality in a Critical Period, as predicted by H4. Results confirming the popularity of viewing a budget suggest that expenditure is correlated with QoS and not the absolute magnitude of price:

'The information that tells me about how much my spending is going down is the most useful...I don't understand about the charge for that bit or this bit, all that I think we really should know is how we're spending in total'.

'No, I don't feel good about seeing that blue representation of my money. How do I know the pace it's going down? I don't want to have to think so much about it'.

Users' evaluations of expenditure suggest that they make dynamic assessments concerning the value of an interaction, as a whole, against their assessment of its price, meaning that users' think about QoS as attached to an entire interaction. Charging schemes might therefore be designed to attach or estimate the cost of an interaction before that interaction starts, in order to provide accurate data to fit users' expectations. Users' assessments of value are therefore reflected by the Commitment they make to reach the goal of their task. This task is represented by the interaction as a whole. 


\section{Conclusions}

The results reported in this paper have shown that:

1) QoS is correlated with expenditure and not absolute price at any one time.

2) Users value predictive feedback over feedback concerned with current network statistics.

3) The Risk Assessment associated with this expenditure depends on the user's goal of interaction.

4) Users have different requirements for feedback although default feedback profiles are feasible.

The work reported in this paper has shown that a vision of future network service must be based on an old principle, that economies are ultimately service-driven. As Peter Drucker (1999) points out:

'Quality in a product or service is not what the supplier puts in. It is what the customer gets out and is willing to pay for. [...] Customers pay only for what is of use to them and gives them value. Nothing else constitutes quality'.

The results reported in this paper have implications for network designers and HCI practitioners. With growing potential to support interactive applications, the real challenge for network designers does not solely lie in maximising utilisation of operations inside the network, but in ensuring that the service provided is both efficient and subjectively valuable to users. However, identifying a trend in behaviour and a cause for that behaviour is not the same thing as being able to predict it. Users' behaviour in response to levels of QoS needs to be predicted by defining the relationship, not only between conceptual factors, but between subjective perceptions and key magnitudes manipulated by the network infrastructure. The combination of grounded theory and experimental methods in this paper provide a framework for conducting HCI research that can, a) describe users' behaviour during interaction and, b) prescribe from conceptual models that represent the motivations for such behaviour. These models have shown that it is not enough to simply configure certain levels of quality to users, it is the interpretation of these figures that must be represented (i.e. the meaning of statistics to the user's current situation). For QoS to be acceptable, users must be able to make an informed decision about their requests, otherwise any valid link between demand and supply is diminished. By applying a combination of methods, this paper has shown what information users require to make such decisions.

We have shown that when users have to pay for the quality they receive, they require dynamic feedback concerning their expenditure. This requires a re-think about how network store and route information to the user; some kind of 'state' in routers is needed to maintain data of the quality likely to be received. Our results suggest how this should work. For example, we have been able to show that semi-intelligent QoS profiles can be implemented that receives pre-set QoS ranges from users and responds only when the quality drops below a certain range. This way, users would not have to constantly re-evaluate the cost of the quality.

Tolerance of QoS, as in the behavioural expression of any preference, is influenced by socio-technical systems that represent the context of that behaviour. These systems 
cannot be directly engineered for. What is possible is to recognise the role of such systems and to apply methods such as grounded theory to understand how their elements relate to each other. For example, this research has shown that users apply the principle of risk aversion when making decisions about the acceptability of a charging mechanism. This principle is based on an ability to predict future network conditions using feedback provided at the application level.

\section{Further Work}

There is a parallel in our society where technological and even political developments influence the way we interact with computers and the demand made for services [17]. The nature of demand for QoS is both dynamic and evolving. Further work that investigates network QoS and charging must reflect the evolving nature of that environment. The way users perceive network operations and the quality that they deliver depends on a number of non-static factors, such as the way QoS is marketed and the business model that lies behind the network. As the results reported in this paper represent users' perceptions of QoS they are subject to variation. The use of grounded theory has enabled the models to be explained at various levels of granularity. Thus, while top-level concepts are likely to continue to be relevant, continuing work is needed to test the concepts described in this paper for their relevance to an evolving environment. The aim of the experiments was to prove the efficacy of models constructed through grounded theory to predict users behaviour whilst interacting with networks. This has been shown. A next step would be an attempt to define objectively where users set an upper bound or Critical Threshold on the price they are willing to pay for a certain task, and how feedback showing them their expenditure could influence this figure.

Participants in this study show idiosyncratic behavior. This means that a participant who selected a certain option in the first condition they did was, in many cases, more likely to select the same option in the second condition. This means that users' feedback needs are likely to differ. Further work is needed to investigate the effects of demographics on users' requirements for QoS and the way they prefer to pay for it. Research is also needed to look at the effects of long term use of a priced quality system on users' feedback requests. It is likely that users' expectations of the levels of quality they receive will become more accurate through experience of typical levels. Requirements for feedback may then only be needed in Critical Periods.

\section{References}

1. Cullinane, P.: Ready, Set, Crash. Telephony 3 (1998) 3-13

2. Cocchi, R., Shenker, S., Estrin, D., Zhang, L.: Pricing in Computer Networks: Motivation, Formulation and Example. IEEE/ACM Transactions on Networking 1 (1993) 614-627 
3. Mackie-Mason, J.K., Varian, H.R.: Economic FAQs about the internet. Journal of Economic Perspectives 8 (1994) 75-96

4. Clark, D.: Internet Cost Allocation and Pricing. In: McKnight, L.W., Bailey, J.P. (eds.): Internet Economics. MIT Press (1997)

5. Bruhns, J.: A Short Commentary on Usability in Northern Europe. Still a Long Way to Go. Available at http: //www.webword.com/moving/ global0002.html (2001)

6. Bouch, A., Bhatti, N., Kuchinsky, A.J.: Quality is in the Eye of the Beholder: Meeting Users' Requirements for Internet Quality of Service. Proceedings of CHI'2000 (2000) 297-304

7. Cosgrove, J.G., Linhart, P.B.: Customer Choices Under Local Measured Telephone Service. Public Utilities Fortnightly, 30 (1979) 27-31.

8. Brittan, D.: Spending More and Enjoying it Less? Technical Review 100 (1997) $11-12$

9. Bohn, R., Braun, H.W., Wolff, S.: Mitigating the Coming Internet Crunch: Multiple Service Levels via Precedence. Applied Network Research Technical Report, GA-A21530. University of California (1994)

10. Bouch, A., Sasse, M.A.: It Ain't What You Charge, it's the Way that You do it: A User Perspective of Network QoS and Pricing. Proceedings of IM'99 (Boston MA) (1999a) 639-655

11. Bouch, A., Sasse, M.A.: Network Quality of Service: What do Users Need?. Proceedings of IDC'1999. (Madrid, Spain) (1999b) 78-90

12. Watson, A.., Sasse, M. A.: Evaluating Audio and Video Quality in Low-cost Multimedia Conferencing Systems. Interacting with Computers 8 (1996) 255-275

13. Strauss, A., Corbin, J.: Basics of Qualitative Research: Grounded Theory Procedures and Techniques. $2^{\text {nd }}$ edn. Sage (1997)

14. Adams, A., Sasse, M. A., Lunt, P.: (1997) Making Passwords Secure and Usable. Proceedings of HCI'97 (1997) 1-19

15. Adams, A., Sasse, M. A.: Privacy Issues in Ubiquitous Multimedia Environments: Wake Sleeping Dogs, or Let Them Lie? Proceedings of IFIP'99 (1999) 214-221

16. Bouch, A., Watson, A., Sasse, M.A.:QUASS-A Tool for Measuring the Subjective Quality of Real-time Multimedia Audio and Video. Proceedings HCI'98 (1998) (Sheffield, England) 94-96

17. Anagnostov, M.E., Cuthbert, L., Lyratzis, T.D., Pitts, J.M.: Economic Evaluation of a Mature ATM Network. Journal of Selected Areas in Communications 10 (1991) 1503-1509.

18. Drucker, P.: Management. Butterworth-Heinemann (1999) 